\title{
Multicast Delivery of IPTV Over the Internet
}

\author{
Dane L. Jackson, Raymond A. Hansen, and Anthony H. Smith
}

\begin{abstract}
Television represents one of the great advancements in information delivery. Traditionally, television service has been delivered using dedicated communication methods such as terrestrial and satellite based wireless transmissions and fixed cable based transmissions. Some of these delivery mechanisms have advanced and now provide services including voice and Internet access. Another communication method, traditional telephone service, has greatly improved and expanded to deliver services such as television and Internet access.
\end{abstract}

This convergence of service provides cost savings, allowing providers to utilize existing communication networks to deliver additional services to its customers, often at minimal or zero infrastructure cost. One disadvantage of this method is customer reach is still limited to those with access to dedicated service provider networks. The ability to disengage television service from these dedicated networks and move it to a more ubiquitous network would greatly improve the customer reach of the providers.

The most obvious network choice for a delivery medium is the Internet. Given that television delivery mechanisms have already started the progression towards IPTV, the service is a natural fit. One issue hindering this transition is bandwidth availability. In private delivery networks, the issue of bandwidth availability for IPTV is often combated through the use of IP Multicasting. Considering the Internet is already believed to be bandwidth constrained, the use of multicasting could be deemed a requirement. The following paper will explore current issues with deploying IPTV over the Internet, the use of multicast to combat some of these problems, and the inherent challenges of pushing multicast based IPTV services over the Internet.

Index Terms-IPTV, Internet, Multicast

\section{INTRODUCTION}

SINCE its inception, the Internet has suffered from growing pains. Technologies have been developed and services deployed to the masses well before they were mature enough to be implemented in an ideal manner. There are many ongoing discussions about issues with how the Internet is designed. More than a few researchers argue a complete overhaul of the Internet's core structure is the best way to work around or eliminate these issues. One such issue with the Internet is its lack of a ubiquitous method to efficiently transmit a stream of data from a single source to many recipients.

Manuscript received June 30, 2010.

D. L. Jackson is with Purdue University, West Lafayette, IN 47907 USA (phone: 765-588-3492; e-mail: dljackso@purdue.edu).

R. A. Hansen is with Purdue University, West Lafayette, IN, 47907 USA (phone: 765-494-4600; e-mail: hansenr@purdue.edu).

A. H. Smith is with Purdue University, West Lafayette, IN, 47907 USA (phone: 765-494-4600; e-mail: ahsmith@purdue.edu).
Unicast packet transmission represents the ubiquitous method of communications on the Internet. Every web browsing and instant message sending host requires the ability to receive and transmit unicast packets in order to function. However, despite its universal support, unicast is far from the best method for all communications scenarios. Internet Protocol television (IPTV) represents a prime example of a scenario where unicast, while functional, is arguably the most inefficient delivery method. IPTV differs from other more typical network traffic such as web browsing due to the fact that data is transmitted from a single source and needs to be received by multiple recipients in real time.

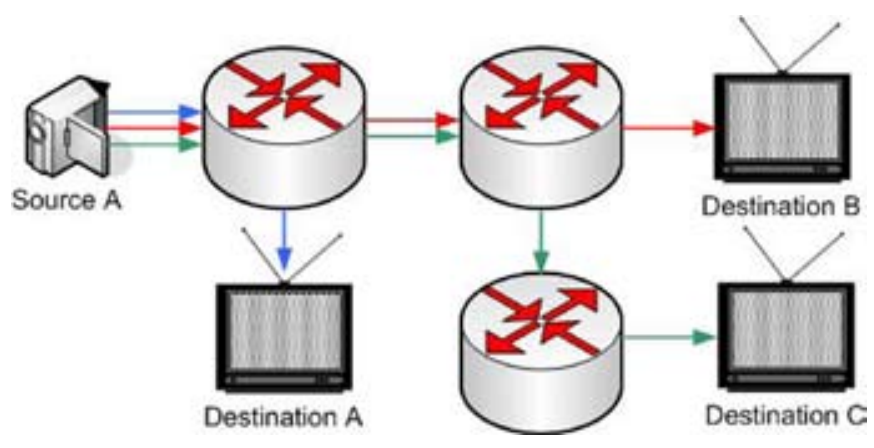

Fig. 1. Unicast Transmission Model

As seen in Figure 1, if done via unicast, each recipient requires its own data stream, causing the source to transmit every packet of data as many times as there are recipients even though they are essentially identical and possibly sharing the same path to multiple recipients. As the number of clients increase, the resulting bandwidth requirements grow at a linear rate proportional to the number of receiving clients.

Because all the clients are receiving the same data, the fact that the source must transmit the same data multiple times is a massive waste of resources. The ability to let routers and switches along the various paths replicate the data as needed in order to reach the desired clients allows for a staggering increase in efficiency. This method of packet transmission is called multicast.

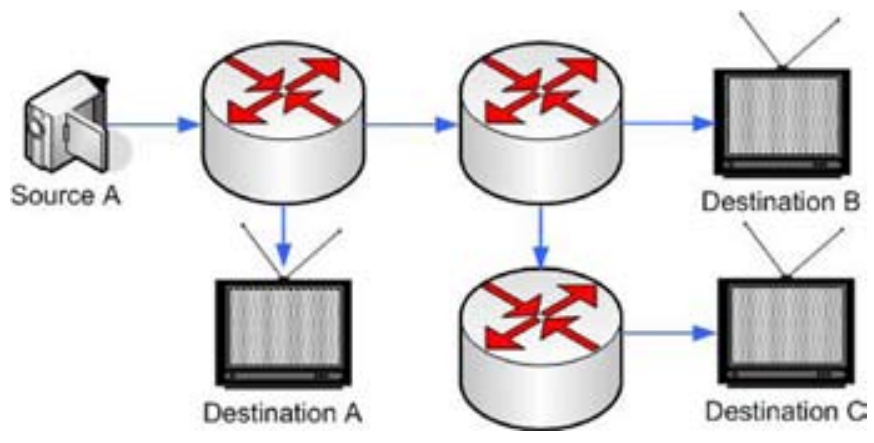

Fig. 2. Multicast Transmission Model 
Once multicast is employed, the amount of bandwidth required by an IPTV stream is drastically reduced. Figure 3 shows the linear growth of bandwidth requirements for a unicast link compared to that of a multicast link.

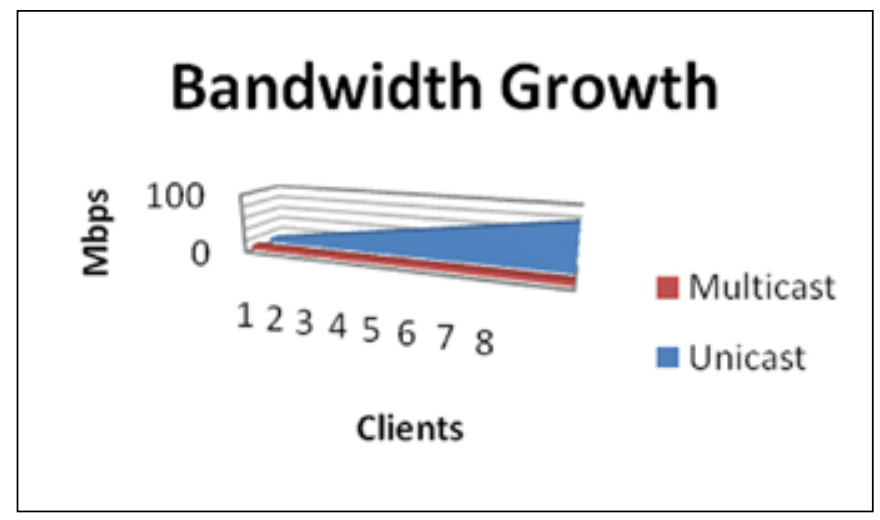

Fig. 3. 8Mbps IPTV Stream Bandwidth Growth

\section{THE REAL WORLD ISSUE}

When an abnormally large amount of recipients flock to a single source, this puts a great amount of strain on the source system, in both bandwidth and CPU resources spent keeping each recipient's data flowing. These Internet "flash mobs" represent a very challenging environment to manage. This challenge is only compounded by the sensitivity of IPTV to an inadequate amount of bandwidth resources.

These flash crowds are referred to as the "The Tiger Effect" by Abor Networks Security Chief Scientist and blogger Craig Labovtiz. This term was coined when several Internet engineers suspected an Internet wide denial-of-service attack was occurring due to an enormous rise in traffic being observed during what was assumed to be a normal day on the Internet. It turned out no attack was occurring. The traffic was identified as Adobe Flash streaming data and the extreme increase was being caused by droves of people flocking to the web site of the U.S. Open in order to watch the IPTV stream of professional golfer Tiger Woods (eventually) win the U.S. Open golf tournament. It is deduced that the majority of people watching from their computers were doing so because the tournament ran over an extra day due to it requiring a playoff match occurring during normal US daytime working hours when viewers were in front of their computers at work rather than in front of their TV at home. This caused portions of the Internet to suffer degraded levels of service (namely the site hosting the video stream and its bandwidth providers). It goes without saying that the service provider was using a unicast based data stream to provide its clients with the video feed.

These flash crowds are almost always generated by sporting, political, or other human interest events. Table 1 contains data from the Akamai Web Usage Index web site representing the top 15 highest peaks in Internet traffic based on peak visitors per minute. Events such as US President Barack Obama's victory speech and inauguration and all of the U.S. College Basketball playoff events offered live IPTV unicast based streams and occurred during typical US business hours.

The issue at hand here is that even though nearly all the visitors were attempting to receive the same IPTV stream of an event, each individual visitor required their own unicast stream causing huge inefficiencies. This results in hosting providers and ISPs (both the bandwidth providers of the hosting site and ISPs of the clients accessing it) either bulking up on available bandwidth in order to sustain the excess load during the short period of time or suffer degraded services (this statement assumes the event is even predictable like the U.S. College Basketball playoffs versus an event of chance as seen with the "Tiger Effect" where the playoff match was not expected). It is noteworthy to point out that the provider hosting CNN's live video stream of the inauguration had to eventually reject new clients attempting to access the IPTV stream due to excess loads. If multicast technology was used to provide these services, hosting sites and consumer ISPs would have barely noticed the effects (if even noticed them at all).

\section{MULTICAST IN THE NETWORK}

Multicast is far from an unsupported or unused protocol. To the contrary, it is used in nearly every local area network (LAN) containing a computer running a current version of any modern operating systems. Many of these operating systems employ neighbor and services discovery technology in order to assist users in finding other computers, available services, and people on the network. However, this is less about bandwidth efficiency and more about providing transparent and configuration free functionality.

When looking at bandwidth availability, a network's local area links are typically much less constrained then its wide area links. However, even with Gigabit and 10Gigabit Ethernet running in the LAN, IPTV can still use multicast to improve the efficiency of getting the video streams out of the network core and to the consumer (it also reduces other resources such as CPU load on the source server).

Below is a basic overview of the protocols available to assist with the implementation of multicast in both LAN and WAN environments. These protocols also span the data link and network layers of the OSI model.

\section{A. IGMP}

Multicast in the LAN is made relatively straight forward through the use of Internet Group Management Protocol (IGMP). This protocol provides the host attempting to join a multicast group the ability to notify adjacent multicast enabled routers of its intent. This notification makes the routers aware of the fact that it needs to start forwarding the desired multicast session to the network in which the IGMP join request was heard.

\footnotetext{
${ }^{1}$ This effect is a close cousin of but differs from the effect known as being "Slashdotted" where visitors are unable to view a web site after being referenced on the front page of the Slashdot web site due to a sudden increase in traffic to an unsupportable level.
} 
TABLE 1

AKAMAI WEB USAGE INDEX CIRCA MARCH 16, 2009

\begin{tabular}{|c|c|c|c|}
\hline Date & Approx Time (EST) & Peak Visitors/min & Compelling New Events \\
\hline Nov. 4, 2008 & 11:00 PM & $8,572,042$ & $\begin{array}{l}\text { Barack Obama is victorious in historic presidential } \\
\text { election }\end{array}$ \\
\hline June 22, 2006 & 12:00 PM & $7,283,584$ & U.S. eliminated by Ghana in World Cup \\
\hline Mar. 20, 2008 & 2:30 PM & $7,008,325$ & $\begin{array}{l}\text { Day One of U.S. College Basketball } 2008 \text { Playoffs } \\
\text { Coverage }\end{array}$ \\
\hline Mar. 20, 2009 & 2:30 PM & $6,337,283$ & $\begin{array}{l}\text { Day Two of U.S. College Basketball } 2009 \text { Playoffs } \\
\text { Coverage }\end{array}$ \\
\hline Mar. 19, 2009 & 4:30 PM & $5,988,459$ & $\begin{array}{l}\text { Day One of U.S. College Basketball } 2009 \text { Playoffs } \\
\text { Coverage }\end{array}$ \\
\hline Mar. 16, 2006 & 3:00 PM & $5,489,918$ & $\begin{array}{l}\text { Day One of U.S. College Basketball } 2006 \text { Playoffs } \\
\text { Coverage }\end{array}$ \\
\hline Jan. 20, 2009 & 11:45 AM & $5,401,250$ & Live coverage and streaming of Obama Inauguration \\
\hline Mar. 15, 2007 & 2:30 PM & $5,100,367$ & $\begin{array}{l}\text { Day One of U.S. College Basketball } 2007 \text { Playoffs } \\
\text { Coverage }\end{array}$ \\
\hline June 16,2008 & 4:30 PM & $4,963,050$ & US Open Championship \\
\hline Jan. 15, 2009 & 5:30 PM & $4,962,523$ & $\begin{array}{l}\text { U.S. Airways Flight } 1549 \text { lands safely in New York's } \\
\text { Hudson River }\end{array}$ \\
\hline Nov. 5, 2008 & 5:00 PM & $4,885,406$ & Post Election Day 2008 coverage \\
\hline Feb. 8, 2007 & 4:30 PM & $4,885,065$ & News breaks on the death of Anna Nicole Smith \\
\hline June 12, 2006 & 2:00 PM & $4,733,201$ & U.S. plays Czech Republic in $1^{\text {st }}$ Round of World Cup \\
\hline Oct. 11, 2006 & 2:45 PM & $4,598,917$ & $\begin{array}{l}\text { Cory Lidle's light plane crashes into New York apartment } \\
\text { building }\end{array}$ \\
\hline Mar. 17, 2006 & 2:30 PM & $4,594,098$ & $\begin{array}{l}\text { Day Two of U.S. College Basketball } 2006 \text { Playoffs } \\
\text { Coverage }\end{array}$ \\
\hline
\end{tabular}

IGMP Snooping is a protocol employed by network switches to listen for and process IGMP packets. This allows the switch to discover which ports are requesting a multicast group and hence control which ports receive the multicast traffic based on this discovery. This process improves LAN performance within individual broadcast domains in a multicast enabled environment by minimizing unnecessary traffic being forwarded to hosts (i.e. controlling broadcast storm).

Router-Port Group Management Protocol (RGMP) is a protocol very similar to IGMP Snooping. It is used by routers in order to determine which networks are participating in a multicast group, allowing the router to prune the unnecessary networks from the multicast tree.

\section{B. PIM}

Protocol Independent Multicast (PIM) provides support for routing multicast traffic between routers within an autonomous system. PIM can function in two flavors, each having advantages and disadvantages. Any Source Multicast (ASM) is the original model employed by PIM. This model provides the functionality to join a multicast group and receive data from any sender in the group. While not well suited for unidirectional applications such as IPTV (though not to exclude the fact that IPTV might one day become a bidirectional service), it functions perfectly for multipoint-to-multipoint video conferencing. Source Specific Multicast (SSM) is the second and newer method. It requires the use of IGMP v3 and adds the functionality for a host to specify a known source to receive multicast data from. This functions much better for unidirectional applications such as IPTV by avoiding undesired multicast "noise" within the group.

Besides ASM and SSM, PIM has two modes it can function in, Dense Mode (DM) and Sparse Mode (SM). In DM, a multicast source is broadcasted to the entire network when it is first brought online. Once this has occurred, nodes will respond with a notification of being uninterested known as a prune message. This mode works very well for extremely dense multicast participating host networks because every host will start out as assumed to be interested and then prune back. SM takes an opposite approach to finding clients. In SM, resources known as Designated Routers (DR) and Rendezvous Point (RP) are used to management group memberships to specific multicast sessions. An initial multicast session will not be forwarded to any nodes until an explicit join request has been forwarded to the RP on 
behalf of the interested host by the DR. This mode functions much better when only a small portion of hosts in a network are interested in the multicast session. Sparse-Dense Mode is a dual function mode that allows the mode, sparse or dense, to be specified per multicast group rather than the entire network having to function in one or the other.

PIM-DM, depending on the amount of multicast traffic present on a network, does not scale well as the network size increases. Its initial broadcast nature can create a great deal of network overhead causing already bandwidth constrained interfaces to become flooded leading to issues such as route flapping. "Channel Surfing" on IPTV can also be a major issue with DM because each channel represents a multicast group and pruning is at times not fast enough to clear out the unneeded streams before the network becomes flooded.

Due to requiring an RP, PIM-SM also suffers from certain scalability issues. In large networks, multiple PIM-SM domains will be required and Multicast Source Discovery Protocol (MSDP) provides the needed functionality to link them back into a single multicast network. This is done by peering MSDP capable routers in each PIM-SM domain. Once this has been done, multicast trees can be extended across domains, enabling inter-domain multicast groups can be established.

\section{MOSPF}

Multicast Open Shortest Path First (MOSPF) is an extension to the commonly used OSPF routing protocol that provides interior gateway protocol (IGP) functionality for routing multicast packets. This protocol can often present a simplistic option to enabling multicast routing by leveraging existing OSPF installations.

Though defined as an IGP, MOSPF can be used to help facilitate multicast routing across extremely large campus, metropolitan, and wide area networks. MOSPF inherits its functionality directly from OSPF. It is a link-state based routing protocol. As clients attempt to join a multicast group, MOSPF utilizing its routing tables in order to build the most efficient multicast path. This path is built on demand.

\section{DVMRP}

Distance Vector Multicast Routing Protocol (DVMRP) is defined by its RFP as an IGP used to connect autonomous systems together and exchange multicast group information. Its base routing functionality and design is derived from RIP and also uses flood and prune mechanisms similar to that of PIM-DM. Another feature of DVMRP is support for interoperability with PIM.

Though defined as an IGP, DVMRP possesses the functionality to build tunnels for transporting multicast datagrams across gateways that do not support multicast routing, creating very large and distributed multicast networks. This functionality is achieved by encapsulating the multicast datagrams into unicast packets containing the source and destination addresses of the tunnel. The non-multicast routers can then handle the packets just like any other traffic. Proper configuration of the tunnel metrics and thresholds along with maximizing path efficiency requires prior investigation into the networks being traversed before choosing a peer.

\section{E. mBGP}

Multiprotocol Boarder Gateway Protocol (mBPG) is an extension to the BGP routing protocol. Though its intent is to add functionality for IPv6 and IPX, it also provides support for the distribution of multicast session information via BGP neighbor associations. Due to its inherent integration with BGP, this makes for an obvious candidate to provide a solution to routing multicast groups across the Internet.

\section{IMPLEMENTATIONS}

Throughout the Internet, three implementations of large scale multicast networks were identified. Below is a list of the multicast enabled networks identified and some details about their implementations.

\section{A. State Networks}

One implementation example of large scale multicast deployments are on several state wide networks. Examples of this can be seen in states like Missouri where a network known as MOREnet has been built to connect schools, public libraries, academic institutions, and other government agencies across the state via a single network. In the state of Indiana, a network called I-Light is used to accomplish nearly the exact same purpose as MOREnet. NCREN is another example of a statewide network connecting academia, government, and libraries running in North Carolina. NCREN is documented as running mBGP across its entire core (along with having complete IPv6 functionality).

\section{B. Mbone}

Multicast functionality on an Internet scale exists via theMbone(standing forMulticastBackbone)network. Though there is little current documentation about Mbone, its functionality is alive and well. Sections of the Mbone utilize several of the protocols mentioned above such as MOSPF and mBGP. However, Mbone was originally started using and still heavily uses the tunnel functionality from DVMRP. This allows sites to establish multicast networks across the Internet without requiring every site wishing to participate to get special access or services from their providers (or setup private links to enabled sites). Many of the state networks use Mbone to provide a multicast interlink with the other networks.

In general, Mbone is similar to the state wide 
networks in that it is primarily used by educational institutions as a method of deploying distance learning which often includes IPTV services. Northwestern University provides access to both CSPAN 1 and 2 video streams via multicast over Mbone.

\section{Internet2}

As was discussed previously in this paper, several researchers argue that a complete redesign of the Internet is needed to in order to correct several inherent issues, such as the lack of multicast support. The Internet2 presents just such an example of this idea being put into practice. Even though this network is being built upon technology providing multi-gigabit network links, multicast represents a pivotal role in the network in order to maintain efficient bandwidth usage as services such as high definition IPTV expand throughout the network.

Internet 2 represent the conglomeration of the vBNS, Abilene, National Lambda Rail (NLR), and other networks into a single structure. Just like with the Internet and the Mbone, Internet2's construction is being led by academia and government.

A working group exists in order to oversee the deployment of multicast technologies on Internet 2 . The network is being built from the ground up with support for mBGP, PIM, and MSDP to provide native multicast functionality across the entire network.

\section{LIMITATIONS AND CHALLENGES}

There are several issues that still reside with the use of multicast based IPTV across the Internet. A primary issue is its penetration (or lack of) into the commercial and home sectors. As can be seen in the implementations section, all the large deployments appear to be stuck within academic and government networks. It can easily be argued that the best things (such as the original Internet) start in academia but the day needs to come when it is pushed to the average end user before IPTV can be effectively deployed to the common user. Because of its open nature, providers may be reluctant to deploy their services over the Internet for fear of losing control of their content.

Another issue that still remains and will likely not be answered by multicast is the explosive growth of video on demand (VoD). Sites such as Hulu, YouTube/Google Video, and others represent a massive drain on the available bandwidth resources of the Internet. Multicast represents the answer to television (i.e. live video) but does not have answer for VoD. Technology such as Anycast being implemented via IPv6 will represent the answer to increasing the bandwidth efficiency of VoD services (this is a discussion best left for a different paper).

The last challenge identified in this paper is the performance concerns of already overloaded switches and routers. When it comes to transporting data packets, bandwidth is not always the metric of concern. Packets per second (PPS) plays a massive roll in the performance of a network. Routers and switches handle unicast packets differently than they handle multicast packets, typically taking longer to handle a multicast packet. This results in a reduced PPS capability. This difference causes a discrepancy in the equipment's ability as services shift from unicast to multicast. Some providers might find their routers struggling to handle the demands of their network even after the inefficiency of unicast based IPTV lifted.

\section{CONCLUSION}

The Internet as it stands today provides an amazing level of access to information. As this level of access is increased and the amount of information grows and shifts from text to video, the Internet must grow and change along with it. Multicast represents a technology with the capacity to help deliver IPTV over the Internet by providing a bandwidth efficient delivery mechanism. The ability to shift a large portion of IPTV from unicast to multicast could possibly provide the needed bandwidth breathing room, allowing television providers the ability to deliver their content to all the corners of the Internet.

Moving live media over to multicast will also help small and large ISPs alike to better normalize their bandwidth utilization. The ability to reduce or eliminate the "Tiger Effect" would greatly assist ISPs in providing a high quality level of service by making bandwidth utilization easier to predict.

\section{ACKNOWLEDGMENT}

The primary author would like to acknowledge and thank co-authors and Professors Raymond Hansen and Anthony Smith for supporting the independent study course which provided the source inspiration for this paper.

\section{REFERENCES}

[1] C. Labovitz. (2008, June 17). The Tiger Effect [Online]. Available: http://asert.arbornetworks.com/2008/06/the-tiger-effect/

[2] C. Labovitz. (2009, June 20). The Great Obama Traffic Flood [Online]. Available: http://asert.arbornetworks.com/2009/01/the-great-obamatraffic-flood/

[3] Akamai - Net Usage Index (2009, March 16). Net Usage Index [Online]. Available: http://www.akamai.com/html/technology/nui/ news/index.html

[4] T. Jacobson (2007, February 1). NCREN, $1^{\text {st }}$ Quarter 2007 [Online]. Available: http://redwidow.mcnc.org/files_static/StateofNCREN2007. pdf

[5] J. Godas, B. Field, A. Bernstein, S. Desai, T. Eckert, and H. Parandekar. IP Multicast in Cable Networks [Online]. Available: http://www. cisco.com/en/US/technologies/tk648/tk828/technologies_case_ study0900aecd802e2ce2.html

[6] K. C. Almeroth, "The Evolution of Multicast: From the MBone to Interdomain Multicast to Internet2 Deployment," IEEE Network, January/February 2000.

[7] A. S. Thyagarajan, S. L. Casner, S. E. Deering (1995, May 10). Making the MBone Real [Online]. Available: http://www. cisco.com/en/US/technologies/tk648/tk828/technologies_case_ study0900aecd802e2ce2.html

[8] D. Waitzman, C. Partridge, and S. Deering (1988, November). Distance Vector Multicast Routing Protocol, RFC 1075 [Online]. Available: http://www.faqs.org/rfcs/rfc1075.html

[9] S. Deering (1989, May). Host Extensions for IP Multicasting, RFC 1112 [Online]. Available: http://www.faqs.org/rfcs/rfc1112.html 
[10] D. Estrin, D. Farinacci, A. Helmy, D. Thaler, S. Deering, M. Handley, V. Jacobson, C. Liu, P. Sharma, and L. Wei (1998, June) Protocol Independent Multicast-Sparse Mode(PIM-SM): Protocol Specification, RFC 2362 [Online]. Available: http://www.faqs.org/rfcs/rfc2362.html

[11] A. Adams, J. Nicholas, and W. Siadak (2005, January). Protocol Independent Multicast - Dense Mode (PIM-DM): Protocol Specification (Revised), RFC 3973 [Online]. Available: http://www. faqs.org/rfcs/rfc3973.html

[12] B. Fenner and D. Meyer, (2003, October). Multicast Source Discovery Protocol (MSDP), RFC 3618 [Online]. Available: http://www.faqs. org/rfcs/rfc3618.html

[13] J. Moy (1994, March) Multicast Extensions to OSPF, RFC 1584 [Online]. Available: http://www.faqs.org/rfcs/rfc1584.html

[14] T. Bates, Y. Rekhter, R. Chandra, and D. Katz, (2000, June) Multiprotocol Extension for BGP-4, RFC 2858 [Online]. Available: http://www.faqs.org/rfcs/rfc2858.html

[15] B. Cain, S.Deering, I. Kouvelas, B. Fenner, and A. Thyagarajan (2002, October) Internet Group Management Protocol, Version 3, RFC 3376, [Online]. Available: http://www.faqs.org/rfcs/rfc3376.html

[16] L. Lao, J. Cui, M. Gerla, and D. Maggiorini, (2005). A Comparative Study of Multicast Protocols: Top, Bottom, or In the Middle? 2005 IEEE.

[17] Research: MOREnet: Technical Support [Online]. Available: http:// www.more.net/technical/research/ip-multicast/

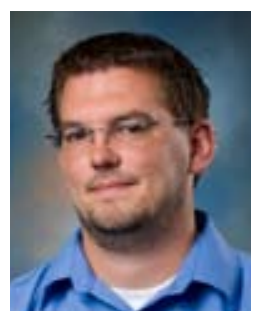

Dane L. Jackson is with the Computer and Information Technology Department and Graduate School at Purdue University. He received a Bachelor of Science degree in Network Engineering Technology in 2004 and is currently pursuing a Master of Science in the same field from Purdue University, West Lafayette, IN, US. He is also currently employed as a Sr. Network Administrator with the Purdue Research Foundation in West Lafayette, IN, US.

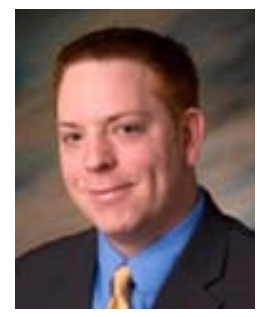

Raymond A. Hansen is an Assistant Professor in the Department of Computer \& Information Technology at Purdue University, where he has taught for the past four years. He currently teaches courses on data communications; architecture, design, construction, security and management of networks; and unified communications. His research interests revolve around the secure integration of real-time services in enterprise networks.

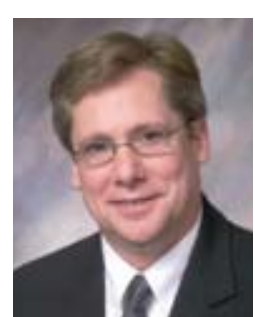

Anthony H. Smith is an Associate Professor with the Computer and Information Technology Department at Purdue University. He has more than 20 years of experience in various technical roles, as well as 15 years experience with computer, network and industrial automation technologies. $\mathrm{He}$ has a degree in Business Administrator from Wittenberg University in 1984. He served in the U.S. Coast Guard for 5 years in a number of technical, management, and law enforcement roles. After leaving the Coast Guard, he pursued a Masters in Technology at Purdue University, which he received in 1994. From 1994 to 2002 Anthony worked in large corporate as well as small business environments serving in project management, technical sales and systems engineering roles. He has designed numerous corporate and municipal networks, as well as leading application development and network engineering groups. Since 2003, Professor Smith has been teaching and conducting applied research in the areas of local and wide-area wireless network design, implementation and security. $\mathrm{He}$ continues to consult on wireless network design projects as well as serving as the Chief Technology Officer for Broadband Antenna Tracking Systems, a Purdue Research Foundation company formed as the result of his patentpending antenna aiming system. 